\title{
Mobile magnetic resonance imaging system provides scans during surgery
}

$\mathrm{N}$ eurosurgeons at the Queen Elizabeth II Health Sciences Centre in Halifax, Nova Scotia, are obtaining magnetic resonance imaging (MRI) scans of patients' brains — while they are operating on them.

The mobile intraoperative imaging system is the first in Canada, although a few Canadian hospitals have high-field MRI systems that can be used during operations. Those, though, require construction of special operating rooms. By contrast, the mobile system is housed in a special closet within the operation room to prevent its magnetic field from interfering with the operation of other equipment.

"This is a low-field MRI that is kept in the operating room at all times," says Dr. Ivar Mendez, a neurosurgeon and chair of the Brain Repair Centre at Queen Elizabeth II.

Currently, the primary use of the PoleStar Surgical MRI System is for removal of brain tumours.

"It allows the surgeon to see clearly where the brain tumour is. More impor-

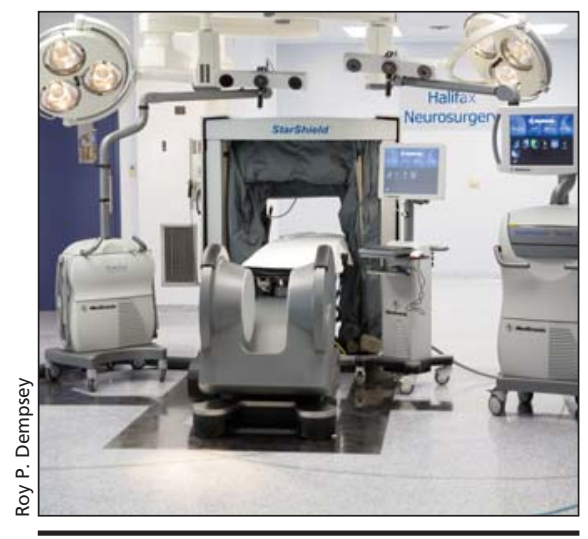

The new Polestar magnetic resonance imaging system at the Queen Elizabeth II Health Sciences Centre in Halifax, Nova Scotia.

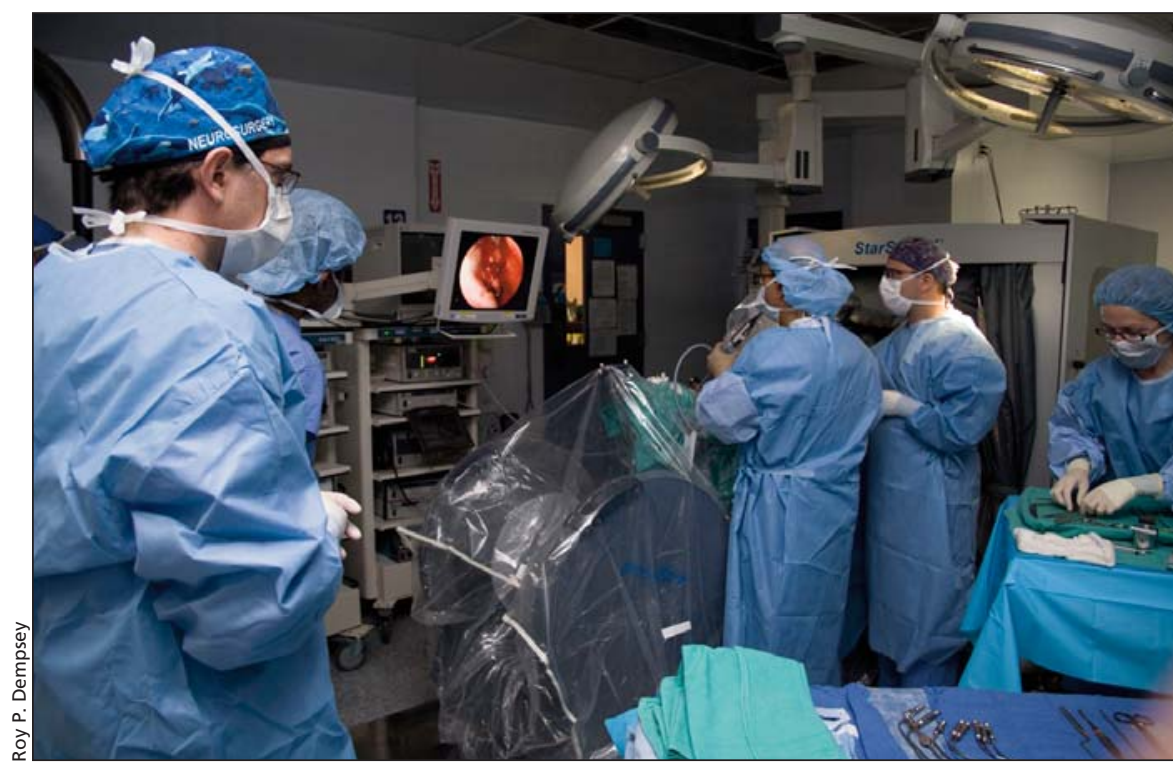

Halifax neurosurgeons say a new mobile intra-operative imaging system allows them to more readily determine if tumors have been completely removed.

tantly, it allows the surgeon to determine if the tumour has been removed completely," says Mendez. "You can see the extent of the resection with the MRI. That is the real value."

In addition, Mendez notes, before physicians close they can do an MRI to ensure there is no post-op brain hemorrhage.

As well, surgery may now be an option for some brain tumour patients previously considered high risk.

The system is also being used for resection of pituitary tumours, a procedure that is done operatively through the patient's nose.

But that's only the tip of the potential for the system, Mendez says. Cell transplantation is another key area for neurosurgery, but the possibilities go well beyond any one medical field. "Intraoperative systems are such a valuable tool, they will become standard in neurosurgery as well as for use in other disciplines."

The system was purchased after the Brain Repair Centre several years ago opted to focus its efforts on 2 key areas: intraoperative imaging and cell therapy.

Surgeons throughout Canada will benefit from the cutting-edge technology, says Dr. Harold Cook, dean of medicine at Dalhousie University in Halifax.

"This new MRI will not only enable Maritime-based neurosurgeons to offer better care to patients, it will be used to train physicians from across the country.

"It will also offer our medical students the chance to experience a medical technology that is not available anywhere else in the country," Cook notes. — Donalee Moulton, Halifax, N.S.

DOI:10.1503/cmaj.091195 